\title{
Results On Generalized Regular And Strongly Regular Near-Rings
}

\author{
SowjanyaMarisetti ${ }^{*}$, Gangadhara rao Ankata ${ }^{2}$, Radharani Tammileti ${ }^{3}$ \\ ${ }^{1}$ Dept. of Mathematics,Eluru College of Engineering \& Technology, Eluru, India. \\ ${ }^{2}$ Dept. of Mathematics, VSR \& NVR College, Tenali, India. \\ ${ }^{3}$ Dept. of Mathematics, Lakireddy Balireddy College of Engineering, Mylavaram, India. \\ sowjanyachallari@gmail.com ${ }^{1}$
}

Article History: Received: 11 January 2021; Accepted: 27 February 2021; Published online: 5 April 2021

\begin{abstract}
Some results on r-regular (r-RN) and also in s-weakly regular (s-WRN)near-rings were established in this article. It is proved that for a near-ring $\mathscr{H} \in \eta_{0}$ is $\mathbf{s}-\mathrm{WRN}$, then $\mathscr{H}$ is simple iff $\mathscr{H}$ is integral. And also proved that for an r-RN $\mathscr{H}$ with unity and satisfies IFP, then $\mathscr{H}$ has the strong IFP iff $\mathscr{H}$ is a PSN.
\end{abstract}

Keywords: s-weakly regular, r-regular, strong IFP, IFP.

\section{INTRODUCTION}

Near-rings, an advanced concept, was highly influenced by the Ring-theory. Von-Neumann regular rings give vital information in the structure theory of rings which was first named by VON-NEUMANN. "Generalization of rings "which are familiar with "Near-rings" plays a major part in the development of Mathematics. Several mathematicians studied and developed various concepts in this area, namely, DheenaP [3], B Elavarasan [4] developed the regularity concept by introducing near-rings $s$-weakly regular and strong IFP. This regularity concept was researched by Mason [7], [8], ReddyYV, and MurthyCVLN [10], Groenewald, and Argac [2].Recently, Wendt Gerhard [13], T Manikantan, and S Ram Kumar [6] researched and established several results.

\section{PRELIMINARIES}

Definition 2.1.1. [9] Let $(\mathscr{H},+,$.$) , a non-empty set is designated as R-NR (Right Near-ring) if$

(i) $\quad \mathscr{H}$ holds the "Group" axioms under addition

(ii) $\quad \mathscr{H}$ holds the "Semigroup" axioms under multiplication

(iii) $\quad(l+t) \cdot p=l \cdot p+t \cdot p$ for all, $1, \mathrm{t}, \mathrm{p} \in \mathscr{H}$ (Right distributive law)

Moreover, we assume that an R-NR is $(\mathscr{H},+,$.$) and we designate it as \mathscr{H}$ except and otherwise mentioned. We write 'lp' to denote 'l.p' for any two elements ' $l$ ' and ' $h$ ' in $\mathscr{H}$. For basic definitions and other related theories, we refer the reader to [9]. We recall the following.

Definition 2.1.2. A near-ring $\mathscr{H}$ is demonstrated as "ZSN (Zero-Symmetric Near-ring)" provided $g o=$ 0 for all $g$ is in $\mathscr{H}$.e., $\mathscr{H}=\mathscr{H}_{0}$.

Example 2.1.3. Let $(\mathscr{H},+)$ where $\mathscr{H}=\{l, t, p, s\}$ be the Klein's four group. Then $(\mathscr{H},+,$.$) represents an$ example for ZSN and expressed it as $\mathscr{H} \in \eta_{0}$. [ 9, p408, (13) $\left.(0,7,13,9)\right]$

Table 1 Addition table

\begin{tabular}{|c|c|c|c|c|}
\hline+ & $l$ & $t$ & $p$ & $s$ \\
\hline$l$ & $l$ & $t$ & $p$ & $s$ \\
\hline$t$ & $t$ & $l$ & $s$ & $p$ \\
\hline$p$ & $p$ & $s$ & $l$ & $t$ \\
\hline$s$ & $s$ & $p$ & $t$ & $l$ \\
\hline
\end{tabular}

Table 2 Product table

\begin{tabular}{|l|l|l|l|l|}
\hline$\cdot$ & $l$ & $t$ & $p$ & $s$ \\
\hline$l$ & $l$ & $l$ & $l$ & $l$ \\
\hline$t$ & $l$ & $t$ & $p$ & $s$ \\
\hline$p$ & $l$ & $l$ & $l$ & $l$ \\
\hline$s$ & $l$ & $t$ & $p$ & $s$ \\
\hline
\end{tabular}

Definition 2.1.4. A subgroup $\wp$ of $\mathscr{H}$ is known as $\mathscr{H}$-subgroup, if $\mathscr{H} \wp \subseteq \wp$.

Definition 2.1.5. An element ' $l$ ' of $\mathscr{H}$ is known as the left identity of $\mathscr{H}$ if $l x=x$ for all $x \in \mathscr{H}$. 
Definition 2.1.6. An element ' $p$ ' of $\mathscr{H}$ is known as the right identity of $\mathscr{H}$ if $y p=y$ for all $y \in \mathscr{H}$.

Definition 2.1.7. An element ' $t$ ' of $\mathscr{H}$ is known as a two-sided identity or an identity element of $\mathscr{H}$ if ' $t$ ' holds both left and right identities in $\mathscr{H}$.

Definition 2.1.8. An element ' $q$ ' of $\mathscr{H}$ is designated as left invertible of $\mathscr{H}$, if there exists an element $b \in \mathscr{H}$ such that $b q=1$. The element ' $b$ ' is referred to as the left inverse of $q$.

Definition 2.1.9. An element ' $s$ ' of $\mathscr{H}$ is designated as right invertible of $\mathscr{H}$, if there exists an element $c \in \mathscr{H}$ such that $s c=1$. The element ' $c$ ' is referred to as the left inverse of $s$.

Definition 2.1.10. An element ' $a$ ' of $\mathscr{H}$ is said to be invertible(unity) of $\mathscr{H}$, if ' $a$ ' satisfies both the definitions 2.1.8 and 2.1.9.

Notation 2.1.11. If $\mathscr{B}, \mathfrak{C} \subseteq \mathscr{H}$ then we can define $\mathscr{B} \mathfrak{C}=\{\mathrm{bc} / b \in \mathscr{B}, c \in \mathscr{H}\}$

Further, we fix the word $\mathscr{H}$ SG to refer to "Normal subgroup ".

Definition 2.1.12.Suppose that $\subseteq$ be a $\mathscr{H S G}$ of $(\mathscr{H},+)$ and is termed as the"Left ideal" of $\mathscr{H}$, provided that $\forall l, p \in$ $\mathscr{H}, \forall s \in \mathbb{S}, l(p+s)-l p \in \mathbb{S}$.

Definition 2.1.13. Suppose that $\subseteq$ be a $\mathscr{H} S \mathrm{SG}$ of $(\mathscr{H},+)$ termed as the "Right ideal" of $\mathscr{H}$ provided that, $\subseteq \mathscr{H} \subseteq \subseteq$.

Definition 2.1.14.Suppose that $\subseteq$ be a $\mathscr{H S G}$ of $(\mathscr{H},+)$ is denoted as an ideal (two-sided ideal)provided that if it follows the conditions both left (right) of $\mathscr{H}$.

Theorem 2.1.15. For a near-ring $\mathscr{H} \in \eta_{0}$, every ideal is a $\mathscr{H}$-subgroup of $\mathscr{H}$.

Definition 2.1.16. Consider a family of left ideals which contains a non-empty subset $\mathscr{H}$ in $\mathscr{H}$. Then the smallest left ideal which is obtained by the intersection of all left ideals containing $\mathscr{F}$ is termed as "left ideal generated by $\mathscr{F}$ "“

Definition 2.1.17.The term" Principal ideal"is referred to as an ideal that is generated by a single element say ' $\mathrm{j}$ ' denoted by $<j>$.

If $\mathfrak{J}$ be a left ideal and is generated by a single element ' $j$ ', then $\mathfrak{J}$ is symbolized by $<j \mid$.

Definition 2.1.18. An element ' $k$ ' is termed as an idempotent of $\mathscr{H}$ if $k^{2}=k$, for $k \in \mathscr{H}$.

Definition 2.1.19. A zero divisor of $\mathscr{H}$ is a component $f \neq 0$ of $\mathscr{H}$ which satisfies $f t=0$ for some nonzero ' $t$ ' in $\mathscr{H}$.

Definition 2.1.20. Let $\mathscr{H}$ is termed to an integral near-ring if it has no non-zero divisors.

Definition 2.1.21. Let $\mathscr{H}$ is termed to a simple near-ring if $\mathscr{H}$ is not having non-trivial ideals.

Definition 2.1.22. Let $\Delta$ be a subset of a $\mathscr{H}$. Then the set $(\mathbf{0}: \Delta)=\{\boldsymbol{h} \in \boldsymbol{N} / \boldsymbol{h} \boldsymbol{x}=\mathbf{0}$, for all $\boldsymbol{x} \in \Delta\}$ is called the annihilator of $\Delta$.

Note 2.1.23. If $\Delta=\{\delta\}$, then $(0: \Delta)$ is denoted by $(0: \delta)$.

Theorem 2.1.24. For any $\delta \in \mathscr{H},(0: \delta)$ is a left ideal of $\mathscr{H}$.

Definition 2.1.25. Let $\mathscr{H}$ is referredto as" Insertion of Factors Property (in short, IFP)", assuming that $\mathrm{jb}=0 \Rightarrow$ $\mathrm{jpb}=0, \forall \mathrm{j}, \mathrm{b}, \mathrm{p} \in \mathscr{H}$.

Theorem 2.1.26. The following conditions are equivalent:

(i) $\quad \mathscr{H}$ has the IFP - property.

(ii) $\quad(\mathbf{0}: \mathbf{h})$ is an ideal of $\mathscr{H}, \forall \mathbf{h} \in \mathscr{H}$.

(iii) $\quad(0: \mathfrak{H})$ is an ideal of $\mathscr{H}$, for all subsets $\mathscr{H}$ of $\mathscr{H}$.

Definition 2.1.27. For each element $\mathrm{d} \in \mathscr{H}$, if $\mathrm{d}^{2}=0 \Rightarrow \mathrm{d}=0$, then $\mathscr{H}$ is referred as reduced near-ring.

Theorem 2.1.28. For each element $k$, $l$ in reduced near-ring, $\mathscr{H} \in \eta_{0}$, then $k l h=k h l$ where $h^{2}=h, h$ is in $\mathscr{H}$ Definition 2.1.29. For each elementl $\in \mathscr{H}$, if $\mathcal{H} l=\mathscr{H} l^{2}$ then $\mathscr{H}$ is termed as" left bi potent".

Definition 2.1.30. For each elementc $\in \mathscr{H}$, there is anelement 1 in $\mathscr{H}$ such that $\mathrm{c}=\mathrm{clc}$, then $\mathscr{H}$ is called as "regular near-ring $(\mathrm{RN})$ ".

Example 2.1.31. Let $\mathscr{H}=\{0, \mathrm{a}, \mathrm{b}, \mathrm{c}\}$ be Klein's four group under addition and multiplication tables $3 \& 4$ as follows.

Table 3 Addition table

\begin{tabular}{|c|c|c|c|c|}
\hline+ & 0 & $a$ & $b$ & $c$ \\
\hline 0 & 0 & $a$ & $b$ & $c$ \\
\hline$a$ & $a$ & 0 & $c$ & $b$ \\
\hline$b$ & $b$ & $c$ & 0 & $a$ \\
\hline$c$ & $c$ & $b$ & $a$ & 0 \\
\hline
\end{tabular}

Table 4 Product table

\begin{tabular}{|c|c|c|c|c|}
\hline$\cdot$ & 0 & $a$ & $b$ & $c$ \\
\hline 0 & 0 & 0 & 0 & 0 \\
\hline
\end{tabular}


Then $(\mathscr{H},+,$.$) is an example for RN.$

\begin{tabular}{|c|c|c|c|c|}
$a$ & $a$ & $a$ & $a$ & $a$ \\
\hline$b$ & 0 & $a$ & $b$ & $c$ \\
\hline$c$ & $a$ & 0 & $c$ & $b$ \\
\hline
\end{tabular}

Definition 2.1.32. For each $\mathrm{r} \in \mathscr{H}$, there is component 1 in $\mathscr{H}$ such that $\mathrm{r}=\operatorname{lr}^{2}$, then $\mathscr{H}$ is demonstrated as" left strongly regular near-ring (left SRN)".

Note2.1.33. [9, p288]. Let $\mathscr{H}$ has the strong IFP provided every homomorphic image of $\mathscr{H}$ has IFP.

Note 2.1.34 [10]. $\mathscr{H}$ has strong IFP if and only if for every ideal $\mathscr{L}$ in $\mathscr{H}$ and $f t \in \mathscr{L}$ implies $f p t \in \mathscr{L}$ for every $f, p, t \in \mathscr{H}$.

\section{2. r-REGULAR NEAR-RINGS}

Definition 2.2.1. [11][12] For each element $p \in \mathscr{H}$, there is anelement ' $h$ ' such that $p=p h, h \in<p \mid$, where $\mathrm{h}$ is an idempotent in $\mathscr{H}$ then $\mathscr{H}$ is demonstrated as" r -Regular Near-ring(r-RN)".

Example 2.2.2. Any $\mathrm{RN}$ is an $\mathrm{r}-\mathrm{RN}$ but the converse need not be true.

(i) Let a near-ring $\mathscr{H}$ defined on $\mathrm{Z}_{6}=\{0,1,2,3,4,5\}$ with operations '+'and '?' given below Tables $5 \& 6$ as follows.

Table 5 Addition table

\begin{tabular}{|c|c|c|c|c|c|c|}
\hline+ & 0 & 1 & 2 & 3 & 4 & 5 \\
\hline 0 & 0 & 1 & 2 & 3 & 4 & 5 \\
\hline 1 & 1 & 2 & 3 & 4 & 5 & 0 \\
\hline 2 & 2 & 3 & 4 & 5 & 0 & 1 \\
\hline 3 & 3 & 4 & 5 & 0 & 1 & 2 \\
\hline 4 & 4 & 5 & 0 & 1 & 2 & 3 \\
\hline 5 & 5 & 0 & 1 & 2 & 3 & 4 \\
\hline
\end{tabular}

Table 6 Product table

\begin{tabular}{|l|l|l|l|l|l|l|}
\hline$\cdot$ & 0 & 1 & 2 & 3 & 4 & 5 \\
\hline 0 & 0 & 0 & 0 & 0 & 0 & 0 \\
\hline 1 & 3 & 5 & 5 & 3 & 1 & 1 \\
\hline 2 & 0 & 4 & 4 & 0 & 2 & 2 \\
\hline 3 & 3 & 3 & 3 & 3 & 3 & 3 \\
\hline 4 & 0 & 2 & 2 & 0 & 4 & 4 \\
\hline 5 & 3 & 1 & 1 & 3 & 5 & 5 \\
\hline
\end{tabular}

This near-ring is $\mathrm{RN}$ and also $\mathrm{r}-\mathrm{RN}$.

(ii) Let a near-ring $\mathscr{H}$ defined on $Z_{8}=\{0,1,2,3,4,5,6,7\}$ with addition is modulo 8 and product table is given below Tables 7 .

Table 7 Product table

\begin{tabular}{|r|r|r|r|r|r|r|r|r|}
\hline. & 0 & 7 & 2 & 3 & 4 & 5 & 6 & 7 \\
\hline 0 & 0 & 0 & 0 & 0 & 0 & 0 & 0 & 0 \\
\hline 1 & 0 & 1 & 3 & 3 & 0 & 1 & 1 & 3 \\
\hline 2 & 0 & 2 & 6 & 6 & 0 & 2 & 2 & 6 \\
\hline 3 & 0 & 3 & 1 & 1 & 0 & 3 & 3 & 1 \\
\hline 4 & 0 & 4 & 4 & 4 & 0 & 4 & 4 & 4 \\
\hline 5 & 0 & 5 & 7 & 7 & 0 & 5 & 5 & 7 \\
\hline 6 & 0 & 6 & 2 & 2 & 0 & 6 & 6 & 2 \\
\hline 7 & 0 & 7 & 5 & 5 & 0 & 7 & 7 & 5 \\
\hline
\end{tabular}

The ideals of this near-ring are $\{0\}$ and $\mathscr{H}$ itself.

This near-ring is $r-R N$ but not $\mathrm{RN}$ (For all $\mathrm{x}, 4 \neq 4$.x.4). 
Theorem 2.2.3. [11] If $\mathscr{H}$ is $r-R N$ with unity and has IFP then $q=q l$ implies $q=l q$ where ' $l$ ' is idempotent. Theorem 2.2.4.[11] If $\mathscr{H}$ is $\mathrm{r}$ - regular near-ring with 1 and has IFP then $\mathscr{H}$ is reduced.

Theorem 2.2.5.If $\mathscr{H} \in \eta_{0}$ is $\mathbf{r}$ - regular near-ring with 1 and has IFP then $\mathscr{H}$ has strong IFP.

Proof: Suppose $\mathscr{H} \in \eta_{0}$, r-RN with land has IFP.

Let $\psi: N \rightarrow N^{1}$ be an epimorphism of r-regular near-ring onto near-ring $N^{1}$.

By the definition of r-regular near-ring, $l=l d, d^{2}=d, d \in<l \mid$

Now, $l=l d, d^{2}=d, d \in\langle l| \subset\langle l\rangle$ so that $d \in\langle l\rangle$.

Consider $\psi(l)=\psi(l d)=\psi(l) \psi(d)$,

$$
\psi(d)=\psi(d d)=\psi(d) \psi(d)
$$

$\psi(d) \in \psi<l>\subseteq<\psi<l>>$ which implies $\psi(d) \in<\psi<l>>$.

Thus, we can conclude that the homomorphic image of r-RN is r-RN.

By the supposition, and by using the theorem 2.2.4, $\mathscr{H}$ is reduced.

Let $q b=0$ then $(b q)^{2}=b(q b) q=b o q=0$

Since $\mathscr{H}$ is reduced, we get that $b q=0$

So, we have that, if $q b=0$ then $b q=0----(1)$

Now, if $\psi(q) \psi(b)=0$ implies $\psi(q b)=0$ which implies $\psi(0)=0$ (using (1))

Then $\psi(b) \psi(q)=\psi(b q)=0$

Therefore, if $\psi(q) \psi(b)=0$ then we get $\psi(b) \psi(q)=0---(2)$

Consider, $\psi(q) \psi(b)=0$

Take $\psi(n b) \psi(q)=\psi(n b q)=\psi(n o)=\psi(0)=0$

Using (2), we get that $\psi(q) \psi(n b)=0$ implies $\psi(q n b)=0$ which implies $\psi(q) \psi(n) \psi(b)=0$ for all $\mathrm{n}$ in $\mathscr{H}$. Thus, the homomorphic image of r-RN satisfies IFP.

Hence, $\mathscr{H}$ has a strong IFP.

Definition 2.2.6. A subset $\mathscr{L} \neq \phi$ of $\mathscr{H}$ is called a 'Pseudo Symmetric Subset '(briefly, PSS) of $\mathscr{H}$ if $\forall$ p, $1 \in \mathscr{H}$, pl $\in \mathscr{L}$ implies prl $\in \mathscr{L} \forall \mathrm{r} \in \mathscr{H}$.

Definition 2.2.7. Let $\mathscr{L} \neq \phi$ of $\mathscr{H}$ is a subset which is indicated as a 'Pseudo Symmetric Ideal' (briefly, PSI) of $\mathscr{H}$ if $\mathscr{L}$ is both a pseudo symmetric subset and an ideal of $\mathscr{H}$.

Definition 2.2.8. A 'Pseudo Symmetric Near-ring' (briefly, PSN) is a near-ring $\mathscr{H}$ in which each ideal of $\mathscr{H}$ is pseudo symmetric.

Theorem 2.2.9. For an r-RN $\mathscr{H}$ with IFP and holds unity 1 then $\mathscr{H}$ has the strong IFP iff $\mathscr{H}$ is a PSN.

Proof. By theorem 2.2.5, $\mathscr{H}$ has a strong IFP.

$\Leftrightarrow$ By Proposition 9.2 of [9], for every ideal $\mathscr{L}$ of $\mathscr{H}, \forall \mathrm{p}, \mathrm{k} \in \mathscr{H}$, and pk $\in \mathscr{L}$ implies prk $\in \mathscr{L} \forall \mathrm{r} \in \mathscr{H}$

$\Leftrightarrow$ Every ideal of $\mathscr{H}$ is a PSI of $\mathscr{H}$

$\Leftrightarrow \mathscr{H}$ is a PSN.

\section{3. $s$ - WEAKLY REGULAR NEAR-RINGS}

The notion of the s-weakly regular ring was first originated by V. Gupta [5] in 1984. Later, Dheena [3] introduced the concept of s-weakly regular near-rings. Recently, Abdullah M. Abdul-Jabbar [1] researched and developed some characteristics in s-weakly regular rings, by studying the above theories, we developed some results on s-weakly regular near-rings.

Definition 2.3.1. Let $\mathscr{H}$ be designated as $\boldsymbol{s}$ - weakly regular (s-WRN) if for each $a \in \mathscr{H}, a=x a$, for some $x \in$ $<a^{2}>$.

Example 2.3.2. Assume $\mathscr{H}$ as a near-ring in Klein four group $\{0, \mathrm{a}, \mathrm{b}, \mathrm{c}\}$ with the operations '十'and '.' shown in table 8 \& 9 mentioned below:

Table 8 Addition table

\begin{tabular}{|l|l|l|l|l|}
\hline+ & 0 & $a$ & $b$ & $c$ \\
\hline 0 & 0 & $a$ & $b$ & $c$ \\
\hline$a$ & $a$ & 0 & $c$ & $b$ \\
\hline$b$ & $b$ & $c$ & 0 & $a$ \\
\hline$c$ & $c$ & $b$ & $a$ & 0 \\
\hline
\end{tabular}

Table 9 Product table

\begin{tabular}{|l|l|l|l|l|}
\hline$\cdot$ & 0 & $a$ & $b$ & $c$ \\
\hline 0 & 0 & 0 & 0 & 0 \\
\hline
\end{tabular}




\begin{tabular}{|l|l|l|l|l|}
$a$ & 0 & $b$ & $c$ & $a$ \\
\hline$b$ & 0 & $c$ & $a$ & $b$ \\
\hline$c$ & 0 & $a$ & $b$ & $c$ \\
\hline
\end{tabular}

The ideals and $\mathscr{H}$-subgroups of $\mathscr{H}$ are $\{0\}$ and $\mathscr{H}$ itself. Then $(\mathscr{H},+,$.$) is an example for s-WRN.$

Theorem 2.3.3: If a near-ring $\mathscr{H} \in \eta_{0}$ is an s-WRN, then $\mathscr{H}$ is reduced near-ring.

Proof: Suppose $q \in \mathscr{H}$ such that $q^{2}=0$.

Since $\mathscr{H}$ is $s$ - weakly regular near-ring, then $q=x q$ for some $x \in\left\langle q^{2}\right\rangle=0$.

So that $q=0$.

Thus $q^{2}=0$ implies $q=0$ for every $q$ in $\mathscr{H}$.

Hence $\mathscr{H}$ is reduced.

THEOREM2.3.4: If a near-ring $\mathscr{H} \in \eta_{0}$ is s-WRN, then $\mathscr{H}$ has IFP.

Proof: Suppose $q b=0,(b q)^{2}=b q b q=b(q b) q=b 0 q=b 0=0$.

By theorem 2.3.3, $\mathscr{H}$ is reduced so that $b q=0$.

There fore if $q b=0$ then $b q=0$.

For all $n \in \mathscr{H},(n b) q=n(b q)=n 0=0 \Rightarrow q n b=q(n b)=0$.

Therefore, $\mathscr{H}$ has IFP.

THEOREM 2.3.5: For a near-ring $\mathscr{H} \in \eta_{0}$ is s-WRN, $\mathscr{H}$ is simple iff $\mathscr{H}$ is integral.

Proof: Suppose $\mathscr{H}$ is simple.

Let $q, b \in \mathscr{H}$ and $q b=0$ and $q \neq 0 \Rightarrow q \in(0: b)$.

By using theorems 2.3.3 and 2.3.4, we have $\mathscr{H}$ is reduced and has IFP. Therefore $(0: b)$ is a two-sided ideal.

Since by our supposition, $\mathscr{H}$ is simple, $(0: b)=\mathscr{H}$.

$b \in \mathscr{H}=(0: b) \Rightarrow b^{2}=0 \Rightarrow b=0$.

Therefore, $\mathscr{H}$ is integral.

Conversely, suppose that $\mathscr{H}$ is integral.

Let $0 \neq \mathrm{I} \unlhd \mathscr{H}, q \neq 0, q \in \mathrm{I}$.

$q=x q, x \in\left\langle q^{2}>\subseteq<q>\subseteq \mathrm{I}\right.$.

$(1-x) q=0 \Rightarrow 1-x=0 \Rightarrow 1=x \in \mathrm{I}$.

Therefore $\mathscr{H}=\mathrm{I}$.

Therefore, $\mathscr{H}$ is simple.

DEFINITION 2.3.6.[2]Let $\mathscr{H}$ is denoted as left quasi duo near-ring (in short, LQD) of $\mathscr{H}$ if every maximal left ideal(M-L-I) of $\mathscr{H}$ is a two-sided ideal.

THEOREM 2.3.7. If a near-ring $\mathscr{H}$ is a LQD having left unity, then $\mathscr{H}$ is s-WRN if and only if $\mathscr{H}=\left\langle\boldsymbol{q}^{2}\right\rangle$ + (0: q) for every $q \in \mathscr{H}$.

Proof: Suppose $\mathscr{H}$ is s-WRN.

Then $q=x q, x \in<q^{2}>\Rightarrow q \in<q^{2}>q$.

$\mathscr{H} q \subseteq \mathscr{H}<q^{2}>q \subseteq<q^{2}>q$ and $<q^{2}>q \subseteq<q>q \subseteq \mathscr{H} q$.

Therefore, $\mathscr{H} q=<q^{2}>q$.

Assume that $\mathscr{H} \neq<q^{2}>+(0: q)$.

Then there is a M-L-I $\mathscr{B}$ such that $\left\langle q^{2}\right\rangle+(0: q) \subseteq \mathscr{B}$.

By the definition of LQD, $\mathscr{B}$ is a two-sided ideal.

Since $q^{2} \in \mathscr{B},<q^{2}>q \subseteq \mathscr{B} q \subseteq \mathscr{H} q=<q^{2}>q$

There exists $f \in<q^{2}>$ such that $(1-f) q=0$.

$\Rightarrow(1-f) \in(0: q)$.

$1=f+(1-f) \in \mathscr{B}$. It is a contradiction.

Therefore $\mathscr{H}=\left\langle q^{2}>+(0: q)\right.$.

Conversely suppose that $\mathscr{H}=<q^{2}>+(0: q)$.

Now, $1 \in \mathscr{H}=\left\langle q^{2}>+(0: q) \Rightarrow 1=t+l, t \in<q^{2}>, l \in(0: q) \Rightarrow l q=0\right.$.

$q=1 q=(t+l) q=t q+l q \Rightarrow q=t q, t \in<q^{2}>$.

Therefore, $\mathscr{H}$ is s-WRN.

Definition 2.3.8. Let $\mathscr{H}$ is designated to strongly reduced if $l \in \mathscr{H}^{2} l^{2} \in \mathscr{H}_{c}$ implies $l \in \mathscr{H}_{c}$.

Note 2.3.9. A near-ring $\mathscr{H}$ is strongly reduced near-ring if and only if for each element $a \in \mathscr{H}$ and any positive integer $n, a^{n} \in \mathscr{H}_{c}$ implies $a \in \mathscr{H}_{c}$

Theorem 2.3.10. For a near-ring $\mathscr{H} \in \eta_{0}$ be an s-WRN, then $\mathscr{H}$ is strongly reduced near-ring.

Proof: By definition of s-WRN, $l=x l, \mathrm{x} \in<l^{2}>$.

If $l^{2} \in \mathscr{H}_{\mathrm{c}}$ implies $<l^{2}>\subseteq \mathscr{H}_{\mathrm{c}}$. 
$l=x l, \mathrm{x} \in<l^{2}>\subseteq \mathscr{H}_{\mathrm{c}}$.

$l \in \mathscr{H}_{\mathrm{c}} \mathscr{H} \subseteq \mathscr{H}_{\mathrm{c}} \Rightarrow l \in \mathscr{H}_{\mathrm{c}}$.

Therefore, $\mathscr{H}$ is strongly reduced near-ring.

\section{CONCLUSIONS}

In this article, we developed some characteristics on $\mathrm{r}-\mathrm{RN}$ and in generalized strongly regular near-rings

\section{ACKNOWLEDGMENTS}

The author wishes a special thanks to the honorable referees fortheir referring to the manuscript and valuable suggestions to improve this publication.

\section{REFERENCES}

1. Abdul-Jabbar AM. (2015) A New Generalization of s-Weakly Regular Rings. Gen. Math. Notes, 27:92106.

2. Argac N, GroenewaldNJ.(2005) Weakly and Strongly Regular Near-rings. Algebra colloq. , 12:121-130.

3. Dheena P. (1989) A generalization of strongly regular near-rings. Indian J. Pure Appl. Math., 20:58-63.

4. Dheena P, Elavarasan B. (2013)On strong ifp near-rings. Int. J. Pure Appl. Math., 87:809-815.

5. Gupta V. (1984) A generalization of strongly regular rings. Acta. Math. Hung., 43:57-61.

6. Manikantan T, RamkumarS.(2020) Pseudo Symmetric Ideals and Pseudo Symmetric Near-rings. Int. J. Math. Comput. Sci.,15:597-609.

7. MasonG. (1980) Strongly regular near-rings. Proc. Edinb. Math. Soc., 23:27-35.

8. MasonG. (1998)A note on strong forms of regularity for near-rings. Indian J. Math., 40:149-153.

9. Pilz G. (1983) Near-rings, North-Holland, Amsterdam.

10. ReddyYV, MurtyCVLN.(1984) On strongly regular near- rings. Proc. Edinb. Math. Soc., 27:61-64.

11. Sowjanya M, Gangadhara Rao A, Anjaneyulu A, Radha Rani T. (2018) r-Regular Near-Rings. International Journal of Engineering Research and Application., 8:11-19.

12. Sowjanya M, Gangadhara Rao A, Radha Rani T,Padmaja V.(2020) Results on r-Regular Near-rings. Int. J. Math. Comput. Sci., 15:1327-1336.

13. WendtG. (2019) Minimal Ideals and Primitivity in Near-rings.Taiwanese J. Math., 23:799-820. 